\title{
Influence of the contact geometry on single walled carbon nanotube/Si photodetector response
}

\author{
M. Scagliotti ${ }^{1}$, M. Salvato ${ }^{1,2 *}$, M. De Crescenzi ${ }^{1,3}$, M. Boscardin $^{4}$ and P. Castrucci $^{1,3}$ \\ ${ }^{1}$ Dipartimento di Fisica, Università di Roma “Tor Vergata”, Via della Ricerca Scientifica 1, I-00133 Rome, Italy \\ ${ }^{2}$ CNR-SPIN Salerno, University of Salerno, Via Giovanni Paolo II 132, I-84084 Fisciano, Italy \\ ${ }^{3}$ INFN, Università di Roma "Tor Vergata", Via della Ricerca Scientifica 1, I-00133 Rome, Italy \\ ${ }^{4}$ Micro-nano Characterization and Fabrication Facility, Fondazione Bruno Kessler (FBK), Via Sommarive 18, I- \\ 38123 Trento, Italy.
}

\begin{abstract}
A systematic study of the optical response of photodetectors based on carbon nanotube/Si heterojunctions is performed by measuring the responsivity, the detectivity and the time response of the devices with different contact configurations. The sensors are obtained by dry transferring single walled carbon nanotube films on the surface of $\mathrm{n}$-doped Si substrate provided with a multifinger contact geometry. The experimental data show a consistent improvement of the photodetector parameters with the increase of the number of fingers without affecting the carbon nanotube film thickness for increase its optical transmittance as in previous experiments. The role of the electrical resistance of the carbon nanotube film is discussed. The obtained results confirm the method and suggest new perspectives in the use of nanostructured materials as part of semiconducting optical devices.
\end{abstract}

\section{Keywords}

Carbon nanotubes; electric contacts; photodetectors; heterojunction

*Corresponding author: Matteo Salvato - matteo.salvato@roma2.infn.it 


\section{Introduction}

Single walled carbon nanotube (SWCNT) thin films present some interesting features as electrical conductivity (Snow et a. 2003; Salvato et al. 2008; De Nicola et al. 2017; Tian et al. 2017) and optical transparency (De Nicola et al. 2017; Blackburn et al. 2008; Tian et al. 2017) which made them suitable for photovoltaic applications over a broad spectral range from the Ultra-Violet (UV) to the Near Infrared (NIR) (De Nicola et al. 2016; Li et al. 2010). Moreover, SWCNT can be easily integrated with conventional silicon electronics to provide hybrid devices with superior characteristics with respect to the constituent materials (An et al. 2013; Wang et al. 2014). With this perspective SWCNT thin films are used as semitransparent conducting window covering the active area of a light sensor representing a competitive alternative to the most commonly used transparent oxides (Tune et al.2014; van de Lagemaat et al. 2006). In fact, the deposition of a SWCNT thin film on a crystalline silicon wafer produces a carbon nanotubes-silicon heterojunction (NSH) where the electric field established at the interface between the two materials acts as the driving field for the photogenerated charges (van de Lagemaat et al. 2006; Jia et al. 2008). Therefore, the whole photodetector (PD) device consists of just two materials, Si and SWCNT film, where the former is the sensitive part where the charges are mainly generated after light absorbing and the latter plays the double role of semitransparent window and charge collector in contact with metallic electrodes of the external circuit (Jia et al. 2008). Since the NSHs can be obtained at room temperature using a dry transfer mechanism for SWCNT deposition on the Si surface (De Nicola et al. 2017), the fabrication process results easy and cheap thus opening new perspectives in reducing costs and saving time with respect to the conventional semiconductor based PD production where multiple steps, at high temperature and in high vacuum conditions, are adopted.

Recently we have presented new PD devices based on NSH operating in the region from near UV to near IR without the use of a power supply, where the SWCNT film thickness was tuned to obtain responsivity $\mathscr{R}$ and detectivity $\mathscr{D}$ values comparable to commercial Si photomultipliers (Salvato et al. 2017). Since the charges are mainly photogenerated inside the Si substrate and collected by the SWCNT film in contact with metallic electrodes, the PD performance depends on the optical transmittance and the electrical resistance of the SWCNT film (Tune et al.2014; Tune et al. 2013). These are two competitive parameters because the increase of the optical transmittance can be obtained by a reduction of the film thickness which implies an increase of its electrical resistance (Blackburn et al. 2008; Tune et al.2014). Nevertheless, the reduction of the thickness of the SWCNT film below a certain value can be detrimental for the homogeneity of the NSH because of the intrinsic porosity of the SWCNT film (Salvato et al. 2017). For these reasons, we investigated the possibility to improve the PD response by acting only on the upper contact configuration and fixing the SWCNT film thickness to an optimal value for optical transmittance. With this purpose, multifinger electrode geometries were specifically designed to enhance the charge collection by the external circuit without increasing the SWCNT thickness and, as a consequence, without reducing its transparency. Multifinger contact geometry was routinely used in CNT based devices for transport measurements (Salvato et al. 2008), field effect study for radiofrequency (Schröter et al. 2013) and detector applications ( $\mathrm{Li}$ et al. 2003). In most of the cases, interdigitated electrodes were designed to better collect the charge carriers which have to be delivered to the external circuit. This is crucial in the case of high sensitive photodetectors where a small number of photogenerated charges have to be collected inside a distance comparable to the charge diffusion length (Sze 2002). Nevertheless, the use of interdigitated electrodes becomes much more useful in the case of printed CNT 
films where the contact resistance between the CNTs and the metallic electrodes is high (Jackson et al. 2009). On this regard, the use of multifinger geometries, instead of a simple two electrode configuration, increases the possibility for collecting electrical charges and improve the device performances.

\section{Materials and Methods}

For the NSH-based PDs, substrates specifically designed and provided by the Fondazione Bruno Kessler (Bagolini et al. 2015) were used. They consist of a gold bottom ohmic contact, an n-doped Si region and terminate with platinum top contacts (figure 1a). The crystalline (100) Si region (150 $\mu \mathrm{m}$ thick) has an electrical resistivity $\rho_{S i}=0.53 \Omega \cdot \mathrm{cm}$ due to $N_{D}=10^{16} \mathrm{~cm}^{-3}$ doping atoms. The top contacts are conceived with a multi-finger geometry consisting of $50 \mu \mathrm{m}$ wide platinum combs, deposited on $300 \mathrm{~nm}$ thick $\mathrm{SiO}_{2}$ template, distant $d$ from each other. As shown in figure $1 \mathrm{~b}$ three different configurations of multi-fingers top contacts have been realized: i) which has 1 finger on one side and two on the other side $\left(d_{i}=950 \mu \mathrm{m}\right)$, ii) with three fingers per side $\left(d_{i i}=450\right.$ $\mu \mathrm{m})$ and iii) with five fingers per side $\left(d_{i i i}=250 \mu \mathrm{m}\right)$. The corresponding active areas, i.e. the top n-Si surface free from the Pt electrodes, are $A_{i}=0.087 \mathrm{~cm}^{2}, A_{i i}=0.084 \mathrm{~cm}^{2}$ and $A_{i i i}=0.079 \mathrm{~cm}^{2}$ respectively.

The SWCNT films fabrication process is detailed elsewhere (De Nicola et al. 2015). A powder consisting of metallic (10\%) and semiconducting (90\%) SWCNTs (Sigma-Aldrich, assay 90\%, diameter 0.7-0.9 nm) was dispersed in an aqueous solution $(26 \mu \mathrm{g} / \mathrm{ml})$ with $2 \%$ concentration of sodium-dodecyl-sulfate (SigmaAldrich, assay>98.5\%) anionic surfactant (SDS). After a sonication process by using distilled water to dissolve the surfactant, the water dispersed SWCNTs were dripped on a mixed cellulose ester filter inserted in a test tube for low vacuum filtration. After the filtration, a SWCNT film covers the cellulose filter surface which is printed on the substrate surface allowing the transfer of the SWCNT film. The thickness, the optical and the electrical properties of the obtained SWCNT films depend on the quantity of aqueous solution dripped on the cellulose filter. Therefore, some SWCNT films were deposited on glass substrates for morphological characterization, optical transmittance and sheet resistance measurements (De Nicola et al. 2015, 2016). For the present study, SWCNT films with an optical transmittance of $49 \%$ at a wavelength of $550 \mathrm{~nm}$ and a sheet resistance $R_{\text {sheet }}=274 \Omega / s q$, measured using the van der Pauw method (van der Pauw 1958), were selected. Using the same transfer method, the selected SWCNT films were deposited on the $\mathrm{Si}$ substrates bridging the fingers and becoming in contact with the n-Si top surface. The NSH is built by the contact between the SWCNT film and the n-Si substrate surface free from the Pt contacts. Figure 1c shows a schematic band diagram of the NSH which is assumed to be of Schottky type as demonstrated in (Salvato et a. 2017). Electron-hole pairs generated inside n-Si after light absorption diffuse in the depletion region formed at the interface. Here they are decoupled by the junction potential which drives electrons towards the n-Si region and the holes towards the SWCNT films where they are collected by the metallic multifinger contacts. The electrical measurements were performed by connecting the bottom contact to one terminal and one or both sides of the top contacts to the other terminal of a Keithley 2602A digital multimeter/power supply or of a Tektronix TDS 2022C oscilloscope (figure 1a). In this way, each substrate can be used for multiple finger configurations. The substrate i) in figure $1 \mathrm{~b}$, for example, can be used as a 1, 2 or 3 finger configuration if top contacts 1,2 or both are terminated to the external circuit respectively. This allows to use the same SWCNT thickness and the same NSH in a PD terminated with different number of fingers 
$(N)$ for the photogenerated charge collection. A commercial calibrated light emitter diode (LED Kingbright L7113QBC-D, emission spectrum peaked at $\lambda=460 \mathrm{~nm}$ ), has been used as light source.

\section{Results and Discussion}

Electrically, the equivalent circuit of the NSH PD devices can be sketched as an ideal current generator in parallel with a diode (see figure 1d) (Sze 2002). However, to take into account real power dissipation, a series resistance $R_{S}=R_{S W C N T}+R_{C}+R_{S i}$ (Jackson et al. 2009) must be included which originates from the SWCNT film resistance $\left(R_{S W C N T}\right)$, the contact resistance $\left(R_{c}\right)$ between the SWCNT film and the Pt contacts and the Si substrate $\left(R_{S i}\right)$. The PD current-voltage (I-V) characteristic curve is then described by the following equation (Sze 2002):

$$
I=I_{0} \exp \left(e\left(V+I R_{s}\right) /\left(n k_{B} T\right)\right)-I_{S C}
$$

where $I_{0}$ is the diode inverse saturation current, $e$ is the elementary charge, $k_{B}$ is the Boltzmann constant, $T$ the temperature, $n$ is the diode ideality factor and $I_{S C}$ is the short circuit photocurrent which is zero in dark conditions. figure 2 a shows the current density $J(I=J \cdot A)$ crossing the active area $A_{i i}$ vs. the polarization voltage $V$ of the device using the substrate ii) sketched in figure $1 \mathrm{~b}$, once using three fingers as the top contact (full black line), and then using six fingers (full red line), measured in dark condition. The two curves are indistinguishable in the inverse polarization region $(V<0)$ and in direct polarization at least for low voltage. At high positive voltage the curves separate with a high current driven by the device with a larger number of fingers. The series resistance $R_{s}$ can be estimated fitting the experimental data of figure 2a in the high current region (Sze 2002) by using the expression

$$
\frac{I d V}{d I}=\frac{n k_{B} T}{e}-R_{s} I
$$

obtained by eq. (1). The values of $R_{s}$, calculated with this method, are reported in the main panel of figure $2 \mathrm{~b}$ for all the analyzed samples and show a reduction of $R_{s}$ with $N$ down to a value of about $2 \Omega$ for $N=5$, followed by a constant behaviour. This result suggests that the main contribution to $R_{s}$ comes from $R_{S W C N T}$ which is the only term in the expression of $R_{s}$ that is expected to decrease with $N$. Increasing $N$, in fact, the distance between the fingers decreases and, as a consequence, the length of the SWCNT film constrained between two adjacent fingers decreases as well. Since $\boldsymbol{R}_{S W C N T}=\rho_{S W C N T} d / t$ (where $\rho_{S W C N T}$ is the resistivity of the SWCNT film, $t$ its thickness and $\boldsymbol{d}$ the distance between the fingers), the reduction of $\boldsymbol{d}$ implies a reduction of $\boldsymbol{R}_{S W C N T}$. On the contrary, $R_{c}$ is expected to increase with $N$ because more contacts between the SWCNT film and the multifinger electrodes are formed (Jackson et al. 2009). Finally, the last term $R_{S i}=\rho_{S i} d_{S i} / A \approx 0.1 \Omega$ (where $d_{S i}$ is the Si thickness and $A$ the active area of the PD) is independent on $N$. Therefore, the data in figure $2 b$ indicate that the SWCNT film can be made more conductive without affecting its thickness but operating on the contact configuration. Furthermore, the constant behaviour of $R_{S}$ vs. $N$ suggests that a possible increase of $R_{c}$ balances the further decrease of $R_{S W C N T}$ for $N>5$ leaving $R_{S}$ unchanged and limited to a value of few Ohms. In the inset of the same figure, $\mathrm{R}_{\mathrm{s}}$ is reported as a function of the distance $d$ between the electrodes. The linear dependence indicates that $R_{s}$, and as a consequences $R_{S W C N T}$, has an Ohmic behaviour and can be properly considered as a uniform electrical resistance 
as sketched in figure 1c (Jackson et al. 2009). The intercept of the fitting line gives the residual resistance $R_{s}=1.8$ $\Omega$.

These observations suggest that the reduction of $R_{S W C N T}$ as a consequence of the increase of $N$ favours the photogenerated charge collection improving the response of the PDs. In order to confirm this consideration, a measure of the responsivity $\mathscr{R}$, the detectivity $\mathscr{D}$ and the noise equivalent power $\mathscr{C} \mathscr{G} \mathcal{P}$ was performed starting by the expressions (Durkop et al. 2004):

$$
\begin{aligned}
& \mathscr{R}=\frac{I_{S C}-I_{0}}{P_{o p t}} \\
& \mathscr{D}=\sqrt{\frac{A}{2 e I_{0}}} \mathscr{R} \\
& \mathscr{T} \mathscr{O P}=\sqrt{A \cdot B W} \mathscr{D}^{-1}
\end{aligned}
$$

In these expressions $I_{S C} \neq 0$ and $I_{O} \approx 0$ are the short circuit currents of the PD under illumination and in dark conditions respectively, $P_{o p t}$ is the incident light power, $A$ is the active area and $B W$ is the bandwidth of the optical device. $I_{S C}$ present into eq. (3) is measured by acquiring the $I$ - $V$ characteristics for all the PD under illumination. These are reported as dashed lines in the inset of figure $2 \mathrm{a}$ for the PDs obtained using the substrate ii of figure $1 \mathrm{~b}$. The effect of the photogenerated charges is clearly evidenced by the shift of the curves towards higher values (in absolute value) of current and voltage with respect to the dark condition (Sze 2002). Here we emphasize that $J_{S C}$ of the PD with six fingers (red dashed line) is higher (in absolute value) than that of the PD with three fingers (black dashed line) as better evidenced by the blow-up of the data in the same figure. Furthermore, since $J_{S C}$ is measured at $V=0$ all the PD parameters dependent on this quantity are indicative of the non polarized PDs.

$\mathscr{R}$ and $\mathscr{D}$, as given by eq. (3) and (4), are related to the input-output gain and to the ability of the PD to detect small signals respectively. These quantities are measured as a function of $P_{\text {opt }}$ and shown in figures $3 \mathrm{a}$ and $3 \mathrm{~b}$ respectively. For all the samples, values of $\mathscr{R}$ in the range $0.3-0.8 \mathrm{~A} / \mathrm{W}$ and values of $\mathscr{D}$ in the range $3-7 \cdot 10^{13}$ Jones are obtained. These values are comparable to the commercial Si based PDs (see for example Hamamatsu Catalog at http://hamamatsu.com/sp/hq/catalogs_en.html) and are remarkable if one considers that our devices consist of a single junction without any protective layer and the PDs were unbiased $(V=0)$. The data in figures $3 \mathrm{a}$ and $3 b$ also show the interesting feature that both $\mathscr{R}$ and $\mathscr{D}$ increase with $N$. Since the SWCNT film thickness is maintained constant for all the PDs, its transparency remains the same for all the investigated devices. Therefore, the observed increase of $\mathscr{R}$ and $\mathscr{D}$ with $N$ can be ascribed only to the reduced electrical resistance of the SWCNT thin film due to the reduction of the distance between the fingers.

Since $\mathscr{X} \mathscr{G P}$ depends on the $B W$ of the devices, a measure of the time response $\tau_{r}$ was performed by measuring the rise time for all the analysed samples. This was done measuring the time between $10 \%$ and $90 \%$ of the full signal amplitude after on-off light excitation using a LED as light source and recording the output voltage of the PD as a function of time. In the inset of figure $3 \mathrm{c} \tau_{r}$ is reported for one of our PDs with $N=5$ measured using the LED source pulsed at a frequency of $10 \mathrm{kHz}$ with $P_{o p t}=8 \mathrm{~mW} / \mathrm{cm}^{2}$. The measured values of $\tau_{r}$ are shown in the main figure as a function of $N$ and are in the range $7 \mu$ s-10 $\mu$ s for all the investigated samples. The 
$\tau_{r}$ vs. $N$ dependence shows that the larger $N$ the faster is the PD response. The measure of $\tau_{r}$ allows estimating the operation $B W$ by the expression $B W \approx 0.35 / \tau_{r}$ (Sze 2002) which, substituted inside the expression (5), gives the data reported in figure $3 \mathrm{~d}$ as a function of $P_{\text {opt }}$ for all the investigated samples. The experimental data clearly show a reduction of the noise with the increase of $N$.

The measurement of the time response $\tau_{r}$ of the PD allows to make some further considerations about the role of the SWCNT film resistance. In a separate experiment (Salvato et al. 2018) the mobility ( $\mu$ ) of one of the PDs (the one with 5 fingers) was determined by measuring the current between the two top contacts while a gate voltage was applied to the bottom contact (Durkop et al. 2004). The obtained value was $\mu=5 \cdot 10^{5} \mathrm{~cm}^{2} / \mathrm{V} \cdot \mathrm{s}$ which gives a charge diffusion length $L=\sqrt{k_{B} T \mu \tau_{r} / e} \approx 7 \mathrm{~mm}$ (Sze 2002; Durkop et al. 2004). Comparing this value with the thickness $d_{S i}$ of the Si substrate and the distance $d$ between the electrodes, we find $L>d+d_{S i}$ for all the present devices. This result confirms that all the charges collected by the SWCNT film in any point of the PD surface diffuse towards the electrodes. Therefore, $R_{S W C N T}$ behaves as a uniform electrical resistance crossed by the photocurrent as assumed in the PD equivalent circuit (figure 1d). This is a further confirmation of the ohmic behaviour of $R_{S W C N T}$ as already stated considering its linear dependence on $d$.

All the experimental data acquired for $\mathscr{R}, \mathscr{D}, \tau_{r}$ and $\mathscr{N} \mathscr{E P}$ are consistent with the consideration that the

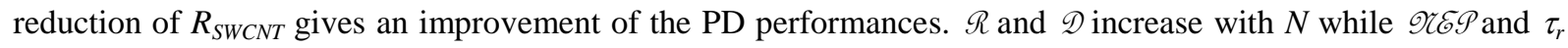
decrease indicating that fast low noise devices with high responsivity and detectivity can be obtained acting on the contact configuration. The improvement of the PD response appears as a direct consequence of the reduction of the distance between the fingers which favours the photogenerated charge collection because of a shorter path to be travelled after reaching the SWCNT film. On this respect, the reduction of the active area, as a consequences of the increase of $N$, seems to affect the PD performances at a lower rate if compared to the enhancement due to the decrease of $R_{S W C N T}$ at least in the range of our investigation. Nevertheless, a threshold should be expected when the fingers completely cover the active area $(N>>10)$ preventing the light absorption in Si substrate.

\section{Conclusion}

In conclusion, the experimental data show that a significant improvement in the PDs response is obtained by increasing the number of contacts. Apart from the high responsivity and high detectivity values obtained, which are comparable to the commercial Si based PDs, the method allowed to collect a greater number of photogenerated charges without operating on the SWCNT film thickness to increase its optical transmittance. This allows to fabricate NSH PDs, maintaining the homogeneity of the junction along the whole active area and opening new perspectives in the use of nanostructured carbon materials for photo and nano-electronic devices. 


\section{References}

An, X.; Liu, F.; Jung, Y.J.; Kar, S. Tunable graphene-silicon heterojunctions for ultrasensitive photodetection. NanoLetters 2013, 13, 909-916. doi.org/10.1021/n1303682j.

Bagolini A et al. 2015 Micromachined silicon radiation sensors: II. Fabrication Technologies Proc. 18th Conf. on Sensors and Microsystems AISEM 2015 (Piscataway, NJ: IEEE) pp 1-4 (XVIII AISEM Annual Conf., (Trento, Italy, 3-5 February 2015).

Blackburn, Jeffrey L.; Barnes, Teresa M.; Beard, Matthew C.; Kim, Yong-Hyun; Tenent, Robert C.; McDonald, Timothy J.; To, Bobby; Coutts, Timothy J.; Heben, Michael J. Transparent conductive single-walled carbon nanotubes networks with precisely tunable ratios of semiconducting and metallic nanotubes. ACSNano 2008, 2 , 1266-1274.

De Nicola, F.; Pintossi, C.; Nanni, F.; Caccioti, I.; Scarselli, M.; Drera, G.; Pagliara, S.; Sangaletti, L.; De Crescenzi, M.; Castrucci, P. Controlling the thickness of carbon nanotube random network films by the estimation of the absorption coefficient. Carbon 2015, 95, 28-33.

De Nicola, F.; Salvato, M.; Cirillo, C.; Crivellari, M.; Boscardin, M.; Scarselli M.; Nanni F.; Cacciotti I.; De Crescenzi, M.; Castrucci, P. Record efficiency of air-stable multi-walled carbon nanotube/silicon solar cells. Carbon 2016, 101, 226-234.

De Nicola, F.; Salvato, M.; Cirillo, C.; Crivellari, M.; Boscardin, M.; Passacantando, M.; Nardone, M.; De Matteis, F.; Motta, N.; De Crescenzi, M.; Castrucci, P. 100\% internal quantum efficiency in polychiral singlewalled carbonnanotube bulk heterojunction/silicon solar cells. Carbon 2017, 114, 402-410.

Durkop, T.; Getty, S.A.; Cobas, E.; Fuhrer, M.S. Extraordinary mobility in semiconducting carbon nanotubes. NanoLetters 2004, 4, 35-39.

Jackson, R.; Graham, S. Specific contact resistance at metal/carbon nanotubes interface. Appl. Phys. Lett. 2009, 94,012109_1-012109_3.

Jia, B.Y.; Jinquan, W.; Wang, K.; Cao, A.; Shu, Q.; Gui, X.; Zhu, Y.; Zhuang, D.; Zhang, G.; Ma, B.; Wang, L.; Liu, W.; Wang, Z.; Luo, J.; Wu, D. Nanotube.silicon heterojunctions solar cells. Advanced Materials 2008, 20, 4594-4598.

Li, J.; Lu, Y.; Ye, Q.; Cinke, M.; Han, J.; Meyyappan, M. Carbon nanotube sensors for gas and organic vapor detection. Nano Letters 2003, 7, 929-933

Koppens, F.H.L.; Mueller, T.; Avouris, Ph.; Ferrari, A.C.; Vitiello, M.S.; Polini, M.; Photodetectors based on graphene, other two dimensional materials and hybrid systems. Nature Nanotechnology 2014, 9, 780-793.

Li, B.X.; Zhu, H.; Wang, K.; Cao, A.; Wei, J.; Li,C.; Jia, Y.; Li, Z.; Li, X.; Wu, D. Graphene-on-silicon Schottky junction solar cells. Advanced Materials 2010, 22, 2743-2748. 
Salvato M.; Cirillo M.; Lucci M.; Orlanducci S.; Ottaviani I.; Terranova M.L.; Toschi F. Charge Transport and Tunneling in Single-Walled Carbon Nanotube Bundles. Phys. Rev. Lett. 2008, 101, 246804_1-246804_4.

Salvato, M.; Scagliotti, M.; De Crescenzi, M.; Castrucci, P. Increasing efficiency in single walled carbon nanotube/n-Si photodetectors by voltage doping. 2018 to be published.

Salvato, M.; Scagliotti, M.; De Crescenzi, M.; Crivellari, M.; Prosposito, P.; Cacciotti, I.; Castrucci, P. Single walled carbon nanotubes/Si heterojunctions for high responsivity photodetectors. Nanotechnology 2017, 28 , 435201_1-435201_9.

Schröeter, M.; Haferlach, M.; Wang, D. Carbon nanotube FET technology for radio-frequency electronics:state-of-the-art overview. IEEE J. of the Electronic devices Society 2013, $1,9$.

Snow E.S.; Novak J.P.; Campbell P.M.; Park D. Random Networks of carbon nanotubes as an electronic material. Appl. Phys. Lett. 2003, 82, 2145-2147.

Sze, S.M. Semiconductor Devices Physics and Technology. John Wiley \& Sons, Inc. USA 2020, ISBN 0-471$33372-7$.

Tian, Y.; Zhang, X.; Geng, H.Z.; Yang, H.J.; Li, C.; Da, S.X.; Wang, J. Jia, S.L. Carbon nanotube/polyurethane films with high transparency, low sheet resistance and strong adhesion for antistatic application RSC Advances 2017, 7, 53018-53024

Tune, D.D.; Shapter, J.G. Effect of nanotubes film thickness on the performance of nanotubes-silicon hybrid solar cells. Nanomaterials 2013, 3, 655-673.

Tune, D.D.; Blanch, A.J.; Krupke, R.; Flavel, B.S.; Shapter, J.G. Nanotube film metallicity and its effect on the performance of carbon nanotubes-silicon cells. Phys. Status Solidi 2014, 211, 1479-1487.

van de Lagemaat, J.; Barnes, T.M.; Rumbles, G.; Shaheen, S.E.; Coutts, T.J.; Weeks, C.; Levitsky, I.; Peltola, J.; Glatkowski, P. Organic solar cells with carbon nanotubes replacing In2O3:Sn as transparent electrode. Appl. Phys. Lett. 2006, 88, 233503_1-233503_3.

van der Pauw, L.J. A method of measuring specific resistivity and Hall effect of disks of arbitrary shape. Philips Res. Rep. 1958 13, 1-9.

Wang, F.; Kozawa, D.; Miyauchi, Y.; Hiraoka, K.; Mouri, S.; Ohno, Y.; Matsuda, K. Fabrication of singlewalled nanotube/Si heterojunction solar cells with high photovoltaic performance. ACS Photonics 2014, 1, 360364. 


\section{Figure Captions}

Fig. 1 a) Schematics of the PD and the measurement system. The $I-V$ measurements were performed polarizing the sample between the bottom and one or both top contacts; b) multifinger geometry top view schematics of the devices: the numbers on each contact indicates the number of fingers used when this contact is connected to the external circuit; c) schematic of the band diagram of the SWCNT/n-Si heterojunction; d) electrical equivalent circuit where the current generator, the diode and $R_{s}$ represent the photogenerated charges, the NHS and the series resistance respectively.

Fig. 2 a) $J-V$ characteristics of two PDs obtained using the same substrate with $N=3$ (black line) and $N=6$ (red line) acquired in dark conditions; inset: $J$ - $V$ characteristics of the same samples acquired in dark condition (full lines, same as the main panel) and under illumination (dashed lines) with $P_{o p t}=100 \mathrm{~mW} / \mathrm{cm}^{2}$; b) series resistance $R_{s}$ vs. the number of fingers $N$ for all the investigated samples. Inset: $R_{s}$ vs. the distance between the fingers. The straight line is a fit to the data.

Fig. 3 a) responsivity and b) detectivity as a function of $P_{o p t}$ for PDs with different $N$; c) rise time vs. $N$ for all the investigated samples. Inset: output voltage of a PD under on-off light excitation. The arrows indicated the $10 \%$ and $90 \%$ of the maximum voltage value for rise time calculation; d) noise equivalent power vs. $P_{o p t}$ for PDs with different $N$. 

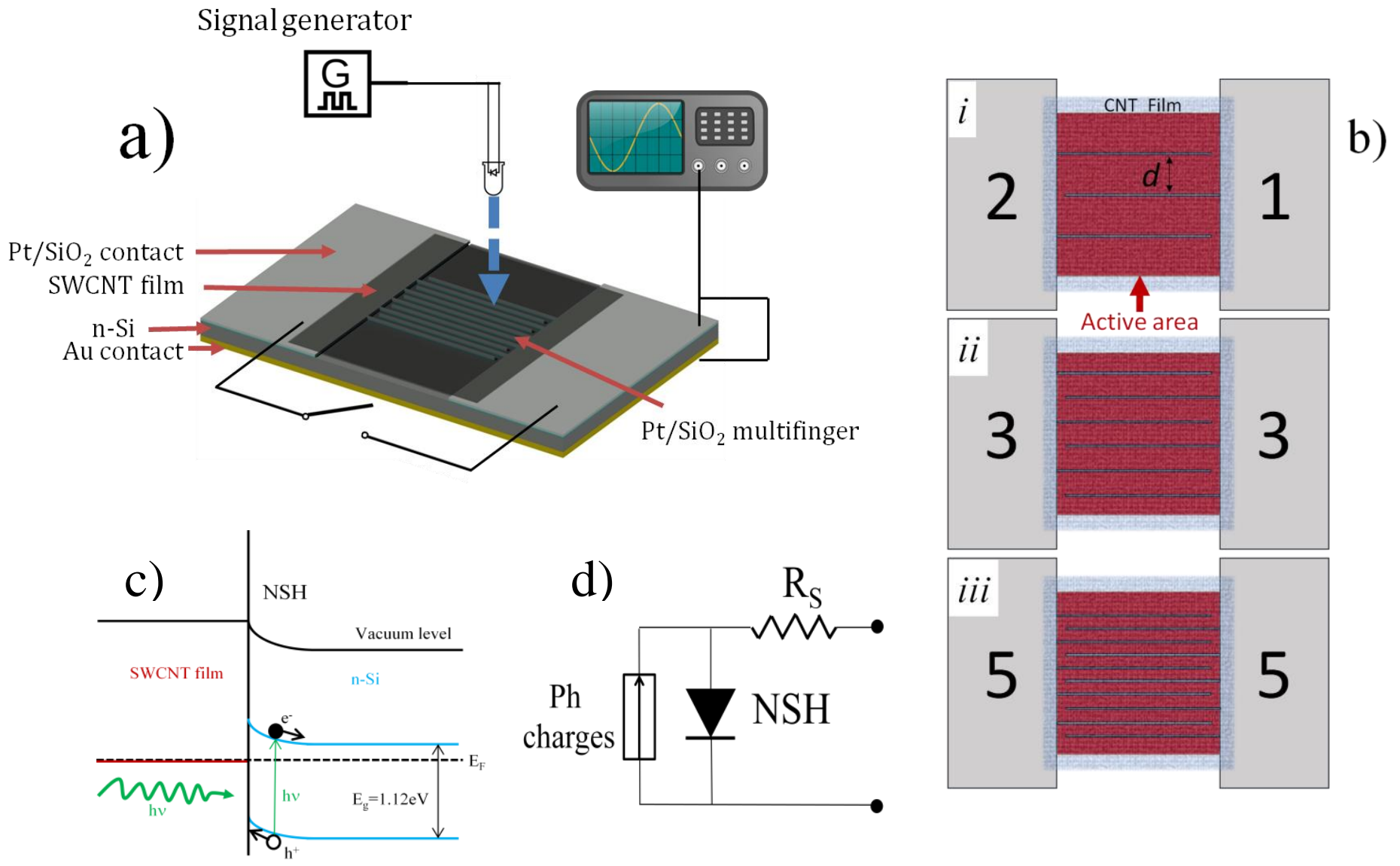

Fig.1 

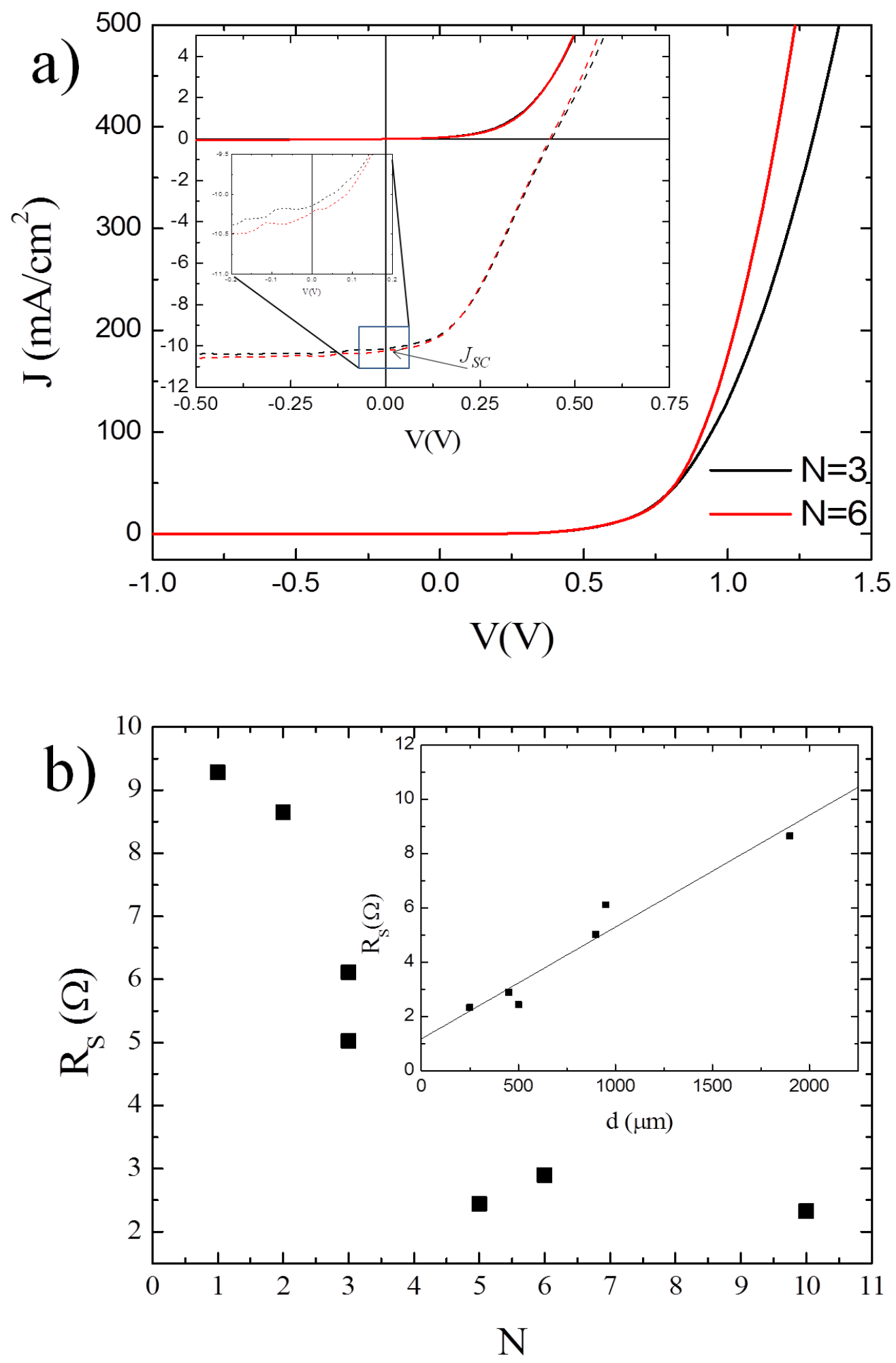

Fig.2 

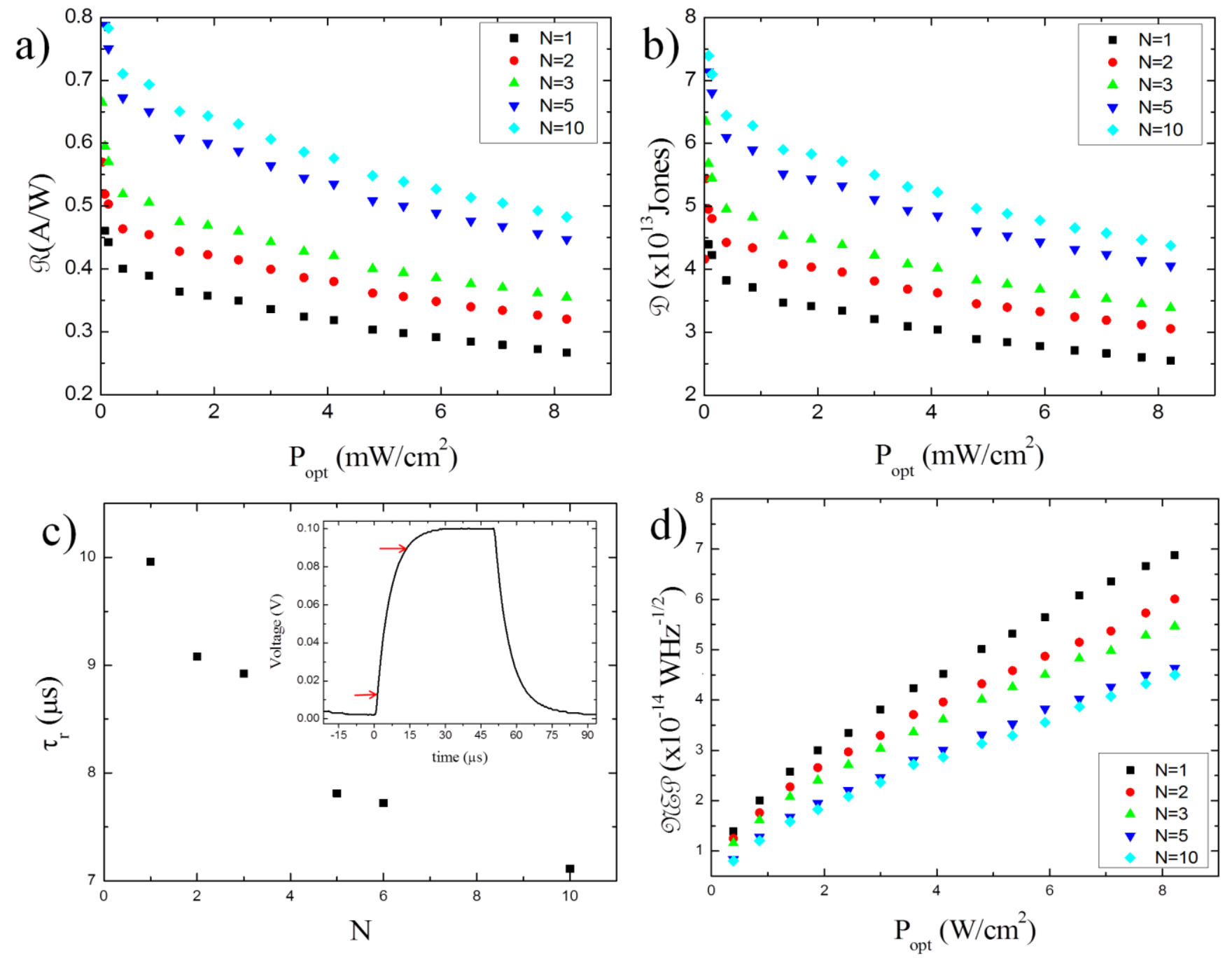

Fig.3 